\title{
CORRESPONDENCE
}

\section{Chlamydophila pneumoniae in asthma}

\section{To the Editors:}

BISCIONE et al. [1] reported a significant association of upper airway Chlamydophila pneumoniae RT-PCR positivity in atopic asthmatics (cumulative rate $22 \%$ ) compared with nonatopic nonasthmatic spouses (9\%). In the discussion, BISCIONE et al. [1] argued in favour of acute, rather than chronic, infection as the explanation for their observation that detection was mostly intermittent rather than persistent. I would like to address three issues raised by this interesting study.

First, in the introduction, BISCIONE et al. [1] stated that C. pneumoniae serology could not differentiate acute from other infections. It is correct to state that serology cannot diagnose chronic infection, but there are established serological criteria for both acute primary and acute secondary infection using the microimmunofluorescence (MIF) test [2]. They also implied that C. pneumoniae serology was nonspecific, i.e. cross-reactive with other Chlamydia species [1], but specificity can be achieved by parallel measurement of other Chlamydia species. For example, the first study they cited as exhibiting these deficiencies was that of HAHN et al. [3], who performed a prospective microbiological and serological study that included acute and convalescent C. pneumoniae MIF serology and was, therefore, able to distinguish serological acute $C$. pneumoniae infection from other serological patterns. In addition, HAHN et al. [3] included species-specific testing for C. trachomatis antibody as a control, and reported that subjects without evidence for an acute C. pneumoniae infection had a strong, statistically significant and specific "dose-response" association of $C$. pneumoniae antibody with wheezing and acute asthmatic bronchitis. We interpreted these serological associations as consistent with either reinfection or chronic infection. Serial MIF testing using acknowledged criteria [2] would have established whether the positive detections reported by BISCIONE et al. [1] were related to acute infection or not.

Secondly, BISCIONE et al. [1] stated (correctly in my opinion) that their data could not distinguish acute infection, reactivation, colonisation or chronic infection. It is unlikely that a $22 \%$ cumulative incidence rate over 3 months was caused by acute exogenous infections because the annual nonepidemic $C$. pneumoniae acute infection rate in the adult population is $<2 \%$ [4]. Acute C. pneumoniae infections can be asymptomatic or associated with only minor respiratory complaints, but a significant minority will cause lower respiratory tract illness [5]. It would be informative to know whether the BISCIONE et al. [1] study was conducted during an epidemic of C. pneumoniae infection in the community, and whether any of the positive detections were associated with an acute respiratory illness.

Thirdly, interpretation of the results is confounded by the mismatch in atopic status introduced by comparing atopic cases with nonatopic controls, i.e. one could argue that atopes are more susceptible to infection than nonatopes, independent of disease status. Future research should include a combination of sensitive nucleic acid detection, serial serological testing, clinical data and appropriate control groups to address the issue of exactly what type of Chlamydophila pneumoniae infection is associated with asthma. Uncertainty about the exact type of infection, however, should not delay performance of clinical trials to establish whether asthma is treatable with antichlamydial antimicrobials.

\section{D.L. Hahn}

Dean Medical Center, Madison, WI, USA.

\section{REFERENCES}

1 Biscione GL, Corne J, Chauhan AJ, Johnston SL. Increased frequency of detection of Chlamydophila pneumoniae in asthma. Eur Respir J 2004; 24: 745-749.

2 Dowell SF, Peeling RW, Boman J, et al. Standardizing Chlamydia pneumoniae assays: recommendations from the Centers for Disease Control and Prevention (USA) and the Laboratory Centre for Disease Control (Canada). Clin Infect Dis 2001; 33: 492-503.

3 Hahn DL, Dodge R, Golubjatnikov R. Association of Chlamydia pneumoniae (strain TWAR) infection with wheezing, asthmatic bronchitis and adult-onset asthma. JAMA 1991; 266: 225-230.

4 Aldous MB, Grayston JT, Wang S-P, Foy HM. Seroepidemiology of Chlamydia pneumoniae TWAR infection in Seattle families, 1966-1979. J Infect Dis 1992; 166: 646-649.

5 Grayston JT. Infections caused by Chlamydia pneumoniae strain TWAR. Clin Infect Dis 1992; 15: 757-761.

\section{From the authors:}

We thank D.L. Hahn for his interest in our study [1] and for commenting upon the interesting issues he raised relating to the study and its interpretation, particularly regarding the presence of acute infection, reactivation, colonisation or chronic infection. These are important issues and informed debate about them is to be welcomed.

D.L. Hahn comments that, in the introduction, we stated that Chlamydophila pneumoniae serology could not differentiate acute from other infections. In fact, we stated that serology cannot reliably differentiate between past and present infection, or acute and chronic infection. We acknowledge that there are published proposed serological criteria for acute primary infection using the microimmunofluorescence (MIF) test [2]. However, these authors stated that "standardized definitions 
for 'acute infection' and 'past exposure' are proposed", and that "these standards should be applied in future investigations and periodically modified as indicated". Although certainly the best advice available to date, we believe that these excellent recommendations are certainly not the last word in C. pneumoniae diagnosis, which remains a very much still evolving field.

As D.L. Hahn states, we also implied that C. pneumoniae serology may be (not "was") nonspecific, i.e. cross-reactive with other Chlamydia species [1]. We stand by this comment, as there is data indicating this may be the case [3]. We do not wish to imply that all or even most $C$. pneumoniae serology is nonspecific, and we believe that most well-performed MIF testing is indeed specific. However, we do believe that more objective, more specific tests are needed to complement MIF in the serodiagnosis of $C$. pneumoniae infection. Thankfully, the number of such available tests is increasing and they are being used in ongoing studies of C. pneumoniae in asthma [4]. We hope that further studies such as these will improve our diagnostic accuracy in due course. We agree with D.L. Hahn that specificity can be improved by parallel measurement of other Chlamydia species and other unrelated organisms, as HAHN et al. [5] and WARK et al. [6] have previously carried out. Such methodological rigour is to be encouraged.

D.L. Hahn states that serial MIF testing using acknowledged criteria [2] would have established whether the positive (PCR) detections we reported [1] were related to acute infection or not. Regrettably, serial blood sampling was not part of our study design, as the original design was conceived to investigate rhinovirus infections [7], for which serology is impractical. The decision to use these samples to investigate the presence of $C$. pneumoniae was only taken once the rhinovirus study was completed. We agree that such serial serological testing would have been desirable and would have added to the evidence, but believe that "established" is too strong a word. The debate regarding C. pneumoniae diagnostic methods will certainly continue until clearly accepted gold standard tests are available. Until such time, the data available can only be interpreted in an unbiased manner, and we strongly support D.L. Hahn's call for future research to include a combination of sensitive nucleic acid detection, serial serological testing and appropriate control groups to address the issue of exactly what type of C. pneumoniae infection is associated with asthma and whether it plays a role in disease pathogenesis.

We thank D.L. Hahn for commenting upon our discussion of whether the PCR detections that we reported were related to acute infection, reactivation, colonisation or chronic infection [1]. We entirely agree that it is unlikely that a $22 \%$ cumulative incidence rate over 3 months was caused by acute exogenous infections because, as D.L. Hahn states, the annual nonepidemic $C$. pneumoniae acute infection rate in the adult population is reported to be $<2 \%$ [8]. To our knowledge, our study was not conducted during a community epidemic of $C$. pneumoniae infection [1]. We are unable to be certain whether any positive detections were associated with an acute respiratory illness, as these samples were taken every 2 weeks, irrespective of the presence or absence of symptoms. This design was adopted to allow us to investigate the frequency of infections independent of bias due to symptom severity, which would have been introduced by studying reported illness episodes $[1,7]$.

We do not believe that our study was able to reliably determine whether these detections were reactivation, colonisation or chronic infections. However, as stated in the discussion, positive detections were all of a duration $<28$ days, except one detection of duration $<42$ days and two detections in which duration could not be defined [1]. The fact that we detected what we believe to be very low bacterial loads, and the short durations of positivity, argue against both acute primary infection and chronic infection. Further studies will be needed, but the working assumption is that these detections relate to colonisation with bacterial loads close to the detection limit of the PCR assay, or to short-term reactivation.

We also agree with D.L. Hahn that our results do not permit us to conclude whether the increased detections relate to atopic status or asthmatic status, as the study design compared atopic asthmatic cases with nonatopic controls. It would clearly be desirable to perform a study including three populations (atopic asthmatic, atopic nonasthmatic and normal nonatopic subjects), in order to answer this question; however, our spouse-pair design, adopted to control for exposure to infectious agents as closely as possible [7], did not allow this.

Finally, we support D.L. Hahn's conclusion that uncertainty about the exact type of infection should not delay performance of clinical trials to establish whether asthma is treatable with antichlamydial antimicrobials. These studies should be highquality, randomised, placebo-controlled studies, and should be supported by further studies on the relationships between Chlamydophila pneumoniae detection and asthma pathogenesis, to help define which populations are most likely to benefit. We believe such studies should initially be focused on populations that are least well served by currently available asthma treatments, namely, acute asthma exacerbations, new onset adult asthma and severe asthma. It is encouraging to note that, in the context of acute exacerbations, where evidence for a role for Chlamydophila pneumoniae infection is increasing $[6,4]$ and where concerns about the induction of antimicrobial resistance to antibiotic therapy would be less worrisome as therapy would be short term, just such a high-quality, randomised, placebo-controlled study is already underway [4].

G.L. Biscione*, J. Corne" ${ }^{\#}$, A.J. Chauhan ${ }^{\circledR}$ and S.L. Johnston ${ }^{+}$ ${ }^{*}$ Centre of Pulmonary Medicine and Rehabilitation, S. Raffaele Hospital, Tosinvest Sanità, Rome, Italy. ${ }^{\#}$ Dept of Respiratory Medicine, Queen's Medical Centre, Nottingham, "The Respiratory Centre, Queen Alexandra Hospital, Portsmouth, and ${ }^{+}$Dept of Respiratory Medicine, National Heart and Lung Institute, Imperial College London, London, UK.

\section{REFERENCES}

1 Biscione GL, Corne J, Chauhan AJ, Johnston SL. Increased frequency of detection of Chlamydophila pneumoniae in asthma. Eur Respir J 2004; 24: 745-749.

2 Dowell SF, Peeling RW, Boman J, et al. Standardizing Chlamydia pneumoniae assays: recommendations from the 
Centers for Disease Control and Prevention (USA) and the Laboratory Centre for Disease Control (Canada). Clin Infect Dis 2001; 33: 492-503.

3 Kern DG, Neill MA, Schachter J. A seroepidemiologic study of Chlamydia pneumoniae in Rhode Island. Evidence of serologic cross-reactivity. Chest 1993; 104: 208-213.

4 Johnston SL, Blasi F, Farrell DJ, Nieman RB. Chlamydia pneumoniae infection in patients with acute exacerbations of asthma: the TELICAST study. Am J Respir Crit Care Med 2004; 169: A586.

5 Hahn DL, Dodge R, Golubjatnikov R. Association of Chlamydia pneumoniae (strain TWAR) infection with wheezing, asthmatic bronchitis and adult-onset asthma. JAMA 1991; 266: 225-230.
6 Wark PA, Johnston SL, Simpson JL, Hensley MJ, Gibson PG. Chlamydia pneumoniae immunoglobulin A reactivation and airway inflammation in acute asthma. Eur Respir J 2002; 20: 834-840.

7 Corne JM, Marshall C, Smith S, et al. Frequency, severity, and duration of rhinovirus infections in asthmatic and non-asthmatic individuals: a longitudinal cohort study. Lancet 2002; 359: 831-834.

8 Aldous MB, Grayston JT, Wang S-P, Foy HM. Seroepidemiology of Chlamydia pneumoniae TWAR infection in Seattle families, 1966-1979. J Infect Dis 1992; 166: 646-649.

\section{Nasal potentials at high altitude}

\section{To the Editors:}

In their recent publication in the European Respiratory Journal, SARTORI et al. [1] explain the difference between their results and previous studies by arguing that in other studies "no particular care was taken to locate the electrode in the inferior turbinate", without contacting the investigators who performed the measurements that they criticise. This argument is not acceptable for us. We can assure them that we paid very careful attention to the placement of the nasal electrode as we are well aware that potentials vary considerably in magnitude in different regions of the nose $[2,3]$. The fact that the magnitude of measured potentials is comparable among all studies at high altitude rules out a significant effect of the site of recording and disproves the argument by SARTORI et al. [1].

We would like to make further observations on the discrepant results which are summarised in table 1 . In a study by MAIRBÄURL et al. [5], performed in 1999 in freezing temperatures and strong winds, subjects reported dryness of the nasal epithelium. This was not a problem in a second study performed in 2003 [6] when weather conditions were warm and nasal dryness was prevented with aerosolised isotonic saline. This manoeuvre entirely prevented the hyperpolarisation of total nasal potential difference. MASON et al. [4] and
SARTORI et al. [1] did not observe a problem with nasal dryness, although the subjects in MASON et al. [4] bathed their nostrils with isotonic saline twice daily. These variations indicate the problems and difficulty of interpretation of nasal potential difference measurements. MAIRBÄURL et al. [5] found no statistically significant change in the amiloride-sensitive change in the nasal potential difference (NP $\Delta$ amil) in highaltitude pulmonary oedema (HAPE)-susceptible subjects; only a nonsignificant trend was reported, whereas, in another study by MAIRBÄURL et al. [6], significantly decreased NP $\Delta$ amil in HAPE was seen, again pointing to possible effects of nasal dryness. This argument is strengthened by the increase seen in the chloride-sensitive change in the nasal potential difference $(\mathrm{NP} \Delta \mathrm{Cl})$ reported by both MAIRBÄURL et al. [5] and MASON et al. [4]. Increased $\mathrm{NP} \Delta \mathrm{Cl}$ is compatible with increased nasal secretion. This possibility was not addressed in the study by SARTORI et al. [1].

In summary, these results indicate that the potential across the nasal epithelium might very well be affected by climatic conditions [7] to which the nose is exposed but to which the alveolar epithelium is not [1,4]. Due to this, particular caution must be exercised when extrapolating data obtained at the nasal epithelium to make claims about changes occurring at the level of the alveolar epithelium.

TABLE 1 Studies reporting the change in nasal potential difference upon ascent to high altitude

\begin{tabular}{|c|c|c|c|c|c|c|}
\hline Change upon ascent to high altitude & Co-NPtot & HAPE-NPtot & Co-NP $\Delta$ amil & HAPE-NP $\Delta$ amil & Co-NPamil-is & HAPE-NPamil-is \\
\hline SARTORI et al. [1] & NS & \# & NS & NS & \# & \# \\
\hline MASON et al. [4] & • & & NS & NS & $\cdot$ & \\
\hline MAIRBÄURL et al. [5] & ・ & • & \# & NS & $\cdot$ & $\bullet$ \\
\hline MAIRBÄURL et al. [6] & & \# & & \# & & \# \\
\hline
\end{tabular}

Co: controls; NPtot: total nasal potential difference; HAPE: high-altitude pulmonary oedema; NPAamil: amiloride-sensitive change in the nasal potential difference; NPAamil-is: amiloride-insensitive nasal potential difference; \#: decreased potential difference, more positive values; " : increased potential difference, more negative values; NS: no significant change. Symbols are shown only when changes were reported to be statistically significant. 